\title{
Young footballers, assessed by deep breathing test, have a higher vagal response
}

\author{
Souad Aboudrar ${ }^{1 *}$, Hanane Rkain ${ }^{1}$, Leila Errguig ${ }^{1}$, Youssouf Radjab ${ }^{1}$, Latéfa Oukerraj ${ }^{2}$, \\ Abdellatif Azzaoui ${ }^{3}$, Azeddine Ibrahimi ${ }^{4}$, Leslie Coghlan ${ }^{5}$, Mohammed Cherti ${ }^{2}$, Halima Benjelloun ${ }^{6}$, \\ Taoufiq Dakka ${ }^{1}$

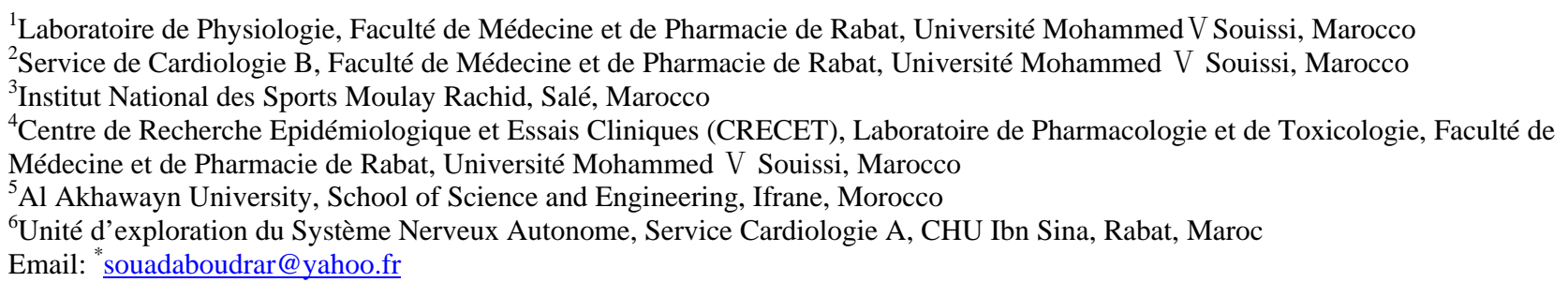

Received 25 April 2012; revised 29 June 2012; accepted 13 July 2012

\begin{abstract}
Parasympathetic function can be assessed by the deep breathing test (DB) as a simple and reproducible cardiovascular reflex. The aim of this study is to use this test to compare the vagal response of a young footballers group to of age-matched untrained normal subjects. Deep breathing test was performed in 2 groups: one of 20 adult young footballers (average age of 19.3 \pm 0.6 years), and a second age-matched group of 20 untrained subjects (average age of $19.6 \pm 0.6$ years). Subjects underwent the DB test after 30 min resting in supine position, and responses were expressed as a percentage of variation of heart rate during the stimulation. Student's $t$-test was used to evaluate statistical differences among the two groups for all parameters (vagal response, heart rate, $\mathrm{VO}_{2 \mathrm{max}} \ldots .$. ) and considering $p<0.05$ as a significant difference. The results showed that vagal response to deep breathing test was significantly higher in the young footballers when compared to the untrained controls $(72.6 \% \pm 16.2 \%$ vs $55.0 \% \pm 12.8 \%$, respectively, $p=0.03$ ). The basal heart rate was significantly lower in footballers than in the controls group $(52.1 \pm 7.4 \mathrm{bat} / \mathrm{min}$ vs $69.8 \pm 14.3$ bat $/ \mathrm{min}, \mathrm{p}<0.01)$. The use of the simple test of deep breathing allowed us to demonstrate that adult young footballers have a significantly lower basal HR and higher parasympathetic response in comparison to untrained subjects.
\end{abstract}

Keywords: Deep Breathing Test; Parasympathetic Response; Autonomic Nervous System; Sudden Death; Trained Footballers

${ }^{*}$ Corresponding author.

\section{INTRODUCTION}

The benefit of physical exercise in controlling cardiovascular risk factors is well established [1,2] and therefore cardiologists widely recommend regular exercise to improve cardiovascular health. It appears important to understand the effects of physical exercise on human physicology. Such studies may help elucidate the fascinating but poorly understood phenomenon of sudden cardiac death in some forms of sport.

Many studies showed that, in the conscious animal model for sudden cardiac death [3], electrical vagal stimulation markedly reduced the incidence of ventricular fibrillation in a high risk subgroup, whereas muscarinic blockade increased malignant arrhythmias in low risk animals. Furthermore, depressed parasympathetic tone is associated with an increased risk of sudden cardiac death since alterations in the neural control of cardiac function contribute to the risk of sudden death. It appears that high vagal response in sport can be harmful and should be assessed thoroughly.

Aerobic training has been suggested to protect the heart against harmful cardiac events by increasing cardiac vagal modulation of heart rate (HR) and also by decreasing sympathetic outflow [4]. It appears that chronic aerobic training alters the autonomic cardiac profile towards parasympathetic predominance, by increased vagal modulation of HR and also, as suggested, by decreased sympathetic activity $[5,6]$. These changes in autonomic cardiac profile can be studied non-invasively using a HR variability technique $[5,6]$ or by invasively measuring sympathetic activity from the peroneal nerve with a microneurography technique [7].

The most common noninvasive techniques used to assess cardiac autonomic changes are the time and frequency 
domain indices of heart rate variability (HRV) and are associated with short-term and long-term endurance exercise training in both leisure sports activity and highperformance training. However, a simpler technique as the deep breathing (DB) test can be used to evaluate the respiratory sinus arrhythmia (RSA) which is accepted as an index of cardiac vagal function.

The aim of this study is to use the DB test to compare the vagal autonomic response of well-trained footballers in comparison to age-matched untrained normal subjects.

\subsection{MATERIALS AND METHODS}

\subsection{Subjects}

We included 20 male footballers (mean age of $19.3 \pm 0.6$ years) who had practiced sport activity on average for more than three years (some of them up to 6 years), and 20 untrained male controls matching them in age.

This human study was approved by the appropriate ethics committee, and all the participants gave their written informed consent prior to their inclusion in the study. Also we have conducted our research ethically according to international standards and as required by the journal and as descibbed by D. J. Harriss and G. Atkinsson [8].

After having their medical history (family, personal and cardiological) carefully recorded, each participant underwent measurement of body mass and height, blood pressure, and heart rate.

Before administrating the maximal oxygen uptake and deep breathing tests, clinical examinations were performed by a recording of 12-lead ECG and echocardiography imaging, to test that all subjects are normal.

\subsection{Maximal Oxygen Uptake: $\mathrm{VO}_{2 \max }$}

Maximal oxygen uptake $\left(\mathrm{VO}_{2 \max }\right)$ was measured in all subjects. $\mathrm{VO}_{2 \max }$ measurement is generally considered as the best indicator of an athlete's cardiovascular fitness and aerobic endurance. In our case, $\mathrm{VO}_{2 \max }$ was obtained from a maximal treadmill test using ergometer cosmed $\mathrm{K} 4$ and it is expressed as milliliters of oxygen used in one minute per kilogram of body weight.

\subsection{Deep Breathing Test Practice}

Deep breathing test was conducted on subjects under no medication in the "service of cardiology A" of the Rabat University Hospital Center Ibn Sina.

Subjects were initially placed in a calm environment in resting conditions. The monitoring of the heart rate (HR) was carried out using a screen (LCD CS 503 E; HELLIGE, EK 512 E).

The DB test evaluated the autonomic function by measuring the modifications of HR in response to a controlled breathing [9]. During the test of DB, the subjects were asked to breathe at the frequency of 6 breathings per minute. The HR was measured by the determination of the interval RR between two adjacent complexes QRS of the EKG, while taking care to exclude from the analysis any aberrant answer like the extra systoles. The vagal response to the $\mathrm{DB}$ is calculated using the following equation: $\mathrm{DB}=\left[\left(\mathrm{RR}_{\operatorname{maxi}}-\mathrm{RR}_{\min i}\right) / \mathrm{RR}_{\min }\right] \times 100$ as previously described in reference [10] with $\mathrm{RR}_{\min }$ obtained at the end of expiration and $\mathrm{RR}_{\max }$ obtained at the end of inspiration.

\subsection{Statistical Analysis}

The quantitative variables were expressed as mean \pm SE and the qualitative variables as manpower and percentages. For each of the parameters, Student's t-test for independent observations was used in order to evaluate statistical differences among the two groups with a $\mathrm{p}$ under 0.05 as an expression of significance.

\section{RESULTS}

\subsection{Descriptive Parameters}

This study included 2 groups, a first one of 20 male footballers practicing sport activity routinely for more than 3 years and a control group of 20, age matched, untrained males. Analysis of different parameters as shown in Table 1, showed no significant differences between the two study groups for age, weight and height.

$\mathrm{VO}_{2 \max }$ was measured in order to determine the subject's aerobic endurance. The footballer group showed a significantly higher maximal oxygen uptake $\left(\mathrm{VO}_{2 \max }\right.$ of $62.6 \pm 7.3 \mathrm{ml} / \mathrm{min} / \mathrm{Kg}$ ) when compared to the control group $\left(\mathrm{VO}_{2 \max }\right.$ of $\left.41.3 \pm 7.4 \mathrm{ml} / \mathrm{min} / \mathrm{Kg}\right)$ demonstrating that this group is well trained with higher endurance.

\subsection{Basal Heart Rate and Blood Pressure}

Blood pressure and the basal heart rate of the two groups were determined to assess heart function and absence of any cardiovascular dysfunction.

As expected, results showed that basal heart rate was significantly lower in footballers group when compared to control group ( $\mathrm{p}<0.01)$. However, there was no significant difference in blood pressure between the 2 groups (Table 2).

Table 1. Groups parameters.

\begin{tabular}{ccccc}
\hline Groups & Age (years) & Weight (Kg) & $\begin{array}{c}\text { Height } \\
(\mathbf{c m})\end{array}$ & $\begin{array}{c}\mathbf{V O}_{2 \max } \\
(\mathbf{m l} / \mathbf{m i n} / \mathbf{K g})\end{array}$ \\
\hline $\mathbf{C}$ & $19.6 \pm 0.6$ & $66.4 \pm 8.9$ & $175.0 \pm 6.4$ & $41.3 \pm 7.4$ \\
$\mathbf{F}$ & $19.3 \pm 0.6$ & $67.5 \pm 6.6$ & $178.2 \pm 4.9$ & $62.6 \pm 7.3$ \\
$\mathbf{p}$ & 0.14 & 0.65 & 0.07 & 0.001 \\
\hline
\end{tabular}

Age (years), weight $(\mathrm{Kg})$, height $(\mathrm{cm})$ and maximal oxygen uptake; $\left(\mathrm{VO}_{2 \max }\right)$ $(\mathrm{ml} / \mathrm{kg})$ values at control $(\mathrm{C})$ and footballers $(\mathrm{F})$. 


\subsection{Vagal Response}

In order to evaluate the vagal response, we measured the modifications of HR in response to a controlled breathing. The results showed that vagal response to deep breathing test was significantly higher in the young footballers when compared to the untrained controls $(72.6 \% \pm 16.2 \%$ vs $55.0 \% \pm 12.8 \%$, respectively, $\mathrm{p}=0.03$ ) (Table 2, Figure 1). The use of the simple test of deep breathing allowed us to demonstrate that adult young footballers have a significantly lower basal HR and higher parasympathetic response in comparison to untrained subjects.

\section{DISCUSSION}

Our study is showing that the use of simple test such as the DB is useful to evaluate the vagal response and may indicate a risk condition for high profile athlete. Changes in heart rate during and recovery from exercise are mediated by the balance between sympathetic and vagal activity. Sympathetic hyperactivity favors the genesis of lifethreatening ventricular tachyarrhythmias whereas vagal activation usually exerts relatively protective and antifibrillatory effects [11].

Deep breathing test exhibited significantly higher vagal response in footballers subjects when compared to untrained ones. It seems that regular aerobic training is accompanied by significant reductions in heart rates both at rest and during submaximal exercise, reflect an increase in autonomic efferent activity and a shift in favor of enhanced vagal modulation of the cardiac rhythm [12].

Table 2. Comparison of basal heart rate, blood pressure and vagal response.

\begin{tabular}{cccc}
\hline Groups & $\begin{array}{c}\text { Basal HR } \\
\text { (b/mn) }\end{array}$ & $\begin{array}{c}\text { Basal BP } \\
\text { (mmHg) }\end{array}$ & $\begin{array}{c}\text { Vagal response } \\
\mathbf{( \% )}\end{array}$ \\
\hline $\mathbf{C}$ & $69.8 \pm 14.3$ & $124.25 \pm 9.8$ & $55.0 \pm 12.8$ \\
$\mathbf{F}$ & $52.1 \pm 7.4$ & $122.27 \pm 8.9$ & $72.6 \pm 16.2$ \\
$\mathbf{p}$ & 0.00 & 0.53 & 0.03 \\
\hline
\end{tabular}

Comparison of basal heart rate (basal HR, b/mn); basal blood pressure (basal $\mathrm{BP}, \mathrm{mmHg}$ ), and vagal response (\%) at control group (C) and footballers (F). $\mathrm{p}<0.05$ considered as significant.

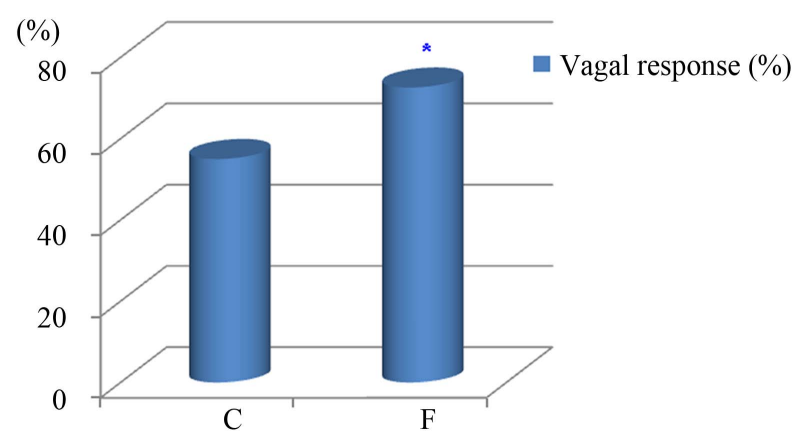

Figure 1. Vagal response assessed by deep breathing test for control group (C) and footballers (F). $\mathrm{p}<0.05$.
Endurance exercise training in healthy human subjects also leads to an increase of heart rate variability (HRV), suggesting increases in vagal tone [13]. It may have beneficial effects on cardiac autonomic activity. In fact, it has been demonstrated that both endurance and team playing athletic activity induce a high parasympathetic tone, suggesting that this type of sports discipline may have a more substantially favorable effect on the cardiac autonomic profile [14]. Furthermore, in the study of the impact of vigorous physical activity on the vagal modulation in young adults, Soares-Miranda L. and Coll. 2009 [15] showed that vagal modulation is enhanced with high levels of physical activity and that it is the number of bouts of vigorous physical activity that is most closely associated with cardiac autonomic nervous system funtion $[15,16]$.

At rest, parasympathetic effects on cardiac electrophysiology have been described in animal [17] and human subjects [18]. The potential mechanism for the protective effect of parasympathetic tone is related to its direct effects on cardiac electrophysiology [19].

Many factors might predispose young athletes to sudden death during sports play as atrial fibrillation which is the most common arrhythmia and has a great impact in morbidity and mortality [20].

As the vagal response increases with sports training, it should probably be dangerous for healthy asymptomatic subjects with spontaneous sinus bradycardia, due to an increase in vagal drive, to practice vigourous training which will enhance their bradycardia and their vagal response to a fatal threshold. Beside the already and justifiable cardiovascular screening with electrocardiography to prevent the risk of sudden cardiac death in young competitive athletes [21-23], we propose the use of deep breathing test, indeed. This test appears to be an easy tool to investigate vagal function integrity in future elite athlete, or before enrolling subjects into an intense training program. However more studies are needed to elucidate the mechanism by which the vagal response is associated to athlete sudden death.

\section{CONCLUSION}

Our study showed that the vagal response was significantly higher in well-trained footballers. However, to characterize the high vagal response, this study would need to be extended to larger groups including older subjects and athlete with longer period of intensive training. These studies need, also, to address the question to which extends this response can switch from being beneficial to a harmful condition particularly for athlete with a very high vagal response leading to increase risks of a sudden cardiac death. 


\section{ACKNOWLEDGEMENTS}

Our thanks to the Ministry of Youth and Sports and to Mr. Brahim Alaoui Belghiti, director of the Moulay Rachid National Institute of Sport (IRFC) for allowing us access to the institute and developing the $\mathrm{VO}_{2 \max }$ technique.

Our thanks also go to Mr. Chefchaouni, director of Ibn Sina University Hospital Center for the management of ecocardiographic examinations.

\section{REFERENCES}

[1] Morris, J.N., Everitt, M.G., Pollard. R., Chave, S.P. and Semmence, A.M. (1980) Vigorous exercise in leisuretime: Protection against coronary heart disease. Lancet, 2, 1207-1210. doi.org/10.1016/0091-7435(84)90037-9

[2] Thompson, P.D., Buchner. D., Pina. I.L., et al. (2003) Exercise and physical activity in the prevention and treatment of atherosclerotic cardiovascular disease. Circulation, 107, 3109-3116. doi:10.1161/01.CIR.0000075572.40158.77

[3] De Ferrari, G.M., Vanoli, E., Curcuruto. P., Tommasini. G. and Schwartz, P.J. (1992) Prevention of life-threatening arrhythmias by pharmacologic stimulation of the muscarinic receptors with oxotremorine. American Heart Journal, 124, 883-890. doi:10.1016/0002-8703(92)90968-2

[4] Billman, G.E. (2002) Aerobic exercise conditioning: A nonpharmacological antiarrhythmic intervention. Journal of Applied Physiology, 92, 446-454.

[5] Akselrod, S., Gordon, D., Madwed, J.B., et al. (1985) Hemodynamic regulation: Investigation by spectral analysis. Journal of Applied Physiology, 249, H867-H875

[6] Akselrod, S., Gordon, D., Ubel, F.A., et al. (1981) Power spectrum analysis of heart rate fluctuation: A quantitative probe of beat-to-beat cardiovascular control. Science, 213, 220-222. doi:10.1126/science.6166045

[7] Delius, W., Hagbarth, K.E., Hongell, A. and Wallin, B.G. (1972) General characteristics of sympathetic activity in human muscle nerves. Acta Physiologica Scandinavica, 84, 65-81. doi:10.1111/j.1748-1716.1972.tb05157.x

[8] Harriss, D.J. and Atkinson, G. (2009) Ethical standards in sport and exercise science research. International Journal of Sports Medicine, 30, 701-702. doi:10.1055/s-0029-1237378

[9] Ewing, D.J., Irving, J.B., Kerr, F., Wildsmith, J.A. and Clarke, B.F. (1974) Cardiovascular responses to sustained handgrip in normal subjects and in patients with diabetes mellitus: A test of autonomic function. Clinical Science \& Molecular Medicine, 46, 295-306.

[10] Aboudrar, S., Benjelloun, H., Benazzouz, A., et al. ( 2007) Evaluation of the vagal activity by the deep-breathing test. Clinical Neurophysiology, 37, 41-46. doi:10.1016/j.neucli.2007.01.005

[11] Airaksinen, K.E. (1999) Autonomic mechanisms and sud- den death after abrupt coronary occlusion. Annals of Medicine, 31, 240-245. doi:10.3109/07853899908995886

[12] Hottenrott, K., Hoos, O. and Esperer, H.D. (2006) Heart rate variability and physical exercise. Current Status, 31, 544-552.

[13] Hull, S.S., Vanoli, E., Adamson, P.B., et al. (1994) Exercise training confers anticipatory protection from sudden death during acute myocardial ischemia. Circulation, 89, 548-552. doi:10.1161/01.CIR.89.2.548

[14] Sztajzel, J., Jung, M., Sievert, K. and De Luna, B.A. (2008) Cardiac autonomic profile in different sports disciplines during all-day activity. The Journal of Sports Medicine and Physical Fitness, 48, 495-501.

[15] Soares-Miranda, L., Sandercock, G., Valente, H., et al. (2009) Vigorous physical activity and vagal modulation in young adults. European Journal of Preventive Cardiology, 16, 705-711. doi:10.1097/HJR.0b013e3283316cd1

[16] Vaseghi, M. and Shivkumar, K. (2008) The role of the autonomic nervous system in sudden cardiac death. Progress in Cardiovascular Diseases, 50, 404-419. doi:10.1016/j.pcad.2008.01.003

[17] Pickoff, A.S. and Stolfi, A. (1990) Modulation of electrophysiological properties of neonatal canine heart by tonic parasympathetic stimulation. American Journal of Physiology, 258, H38-H44.

[18] Prystowsky, E.N., Jackman, W.M., Rinkenberger, R.L., Heger, J.J. and Zipes, D.P. (1981) Effect of autonomic blockade on ventricular refractoriness and atrioventricular nodal conduction in humans. Evidence supporting a direct cholinergic action on ventricular muscle refractoriness. Circulation Research, 49, 511-518. doi:10.1161/01.RES.49.2.511

[19] Rardon, D.P. and Bailey, J.C. (1983) Parasympathetic effects on electrophysiologic properties of cardiac ventricular tissue. Journal of the American College of Cardiology, 2, 1200-1209. doi:10.1016/S0735-1097(83)80351-9

[20] Benjamin, E.J., Wolf, P.A., D’Agostino, R.B., et al. (1998) Impact of atrial fibrillation on the risk of death: The framingham heart study. Circulation, 98, 946-952. doi:10.1161/01.CIR.98.10.946

[21] Corrado, D., Pelliccia, A., Bjornstad, H.H., et al. (2005) Cardiovascular pre-participation screening of young competitive athletes for prevention of sudden death: Proposal for a common European protocol. European Heart Journal, 26, 516-524. doi:10.1093/eurheartj/ehi108

[22] Glover, D.W. and Maron, B.J. (2007) Evolution in the process of screening United States high school studentathletes for cardiovascular disease. American Journal of Cardiology, 100, 1709-1712. doi:10.1016/j.amjcard.2007.07.018

[23] Pelliccia, A. and Corrado, D. (2010) Can electrocardiographic screening prevent sudden death in athletes? British Medical Journal, 341, c4923. doi:10.1136/bmj.c4923 